\title{
Total Serum Protein Predicted Mortality in Patients with St-elevation Myocardial Infarction Who Underwent Primary Percutaneous Coronary Intervention: Results of 8-Year Follow-up
}

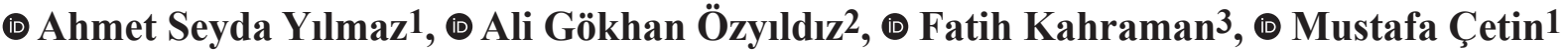

${ }^{1}$ Recep Tayyip Erdoğan University Faculty of Medicine, Department of Cardiology, Rize, Turkey

2Recep Tayyip Erdoğan Training and Research Hospital, Clinic of Cardiology, Rize, Turkey

${ }^{3}$ Kütahya Evliya Çelebi Training and Research Hospital, Clinic of Cardiology, Kütahya, Turkey

\begin{abstract}
Objectives: ST-elevation myocardial infarction (STEMI) is globally one of the leading causes of mortality. Determining modifiable mortality predictors to improve outcomes is critical. Total serum protein (TSP) is a composite indicator of immunity, nutrition, and inflammation and it plays a vital role in biological pathways contributing to cardiovascular diseases. TSP level has not been evaluated in patients with STEMI in the prediction of mortality previously.

Materials and Methods: The patients diagnosed with STEMI between March 2007 and May 2009 were included in the study. TSP was obtained at admission to the hospital. Follow-up period of the study was 8 years and primary endpoint was all-cause mortality. Participants were separated according to the presence of mortality and clinical parameters compared between these two groups.

Results: The mean age of the total 99 patients was $61 \pm 12.4$ years and $82(82.8 \%)$ of them were male. While left ventricular ejection fraction (LVEF) $(\mathrm{p}=0.001)$, serum albumin $(\mathrm{p}=0.014)$, and TSP $(\mathrm{p}<0.001)$ were lower, serum creatinine was
\end{abstract}

Address for Correspondence: Ahmet Seyda Yılmaz, Recep Tayyip Erdoğan University Faculty of Medicine, Department of Cardiology, Rize, Turkey

e-mail: ahmetseydayilmaz@gmail.com ORCID: orcid.org/0000-0003-3864-4023

Received: 10.05.2021 Accepted: 07.06.2021

Cite this article as: Yılmaz AS, Özyıldız AG, Kahraman F, Çetin M. Total Serum Protein Predicted Mortality in Patients with Stelevation Myocardial Infarction Who Underwent Primary Percutaneous Coronary Intervention: Results of 8-Year Follow-up. EJCM 2021;9(2):122-129.

DOI: $10.32596 /$ ejcm.galenos.2021-05-30

${ }^{\circ}$ Copyright 2021 by Heart and Health Foundation of Turkey (TÜSAV) / E Journal of Cardiovascular Medicine published by Galenos Publishing House. 
higher $(\mathrm{p}=0.003)$ in the mortality group. Diabetes mellitus $(\mathrm{p}=0.007)$, increased age $(\mathrm{p}=0.027)$, LVEF $(\mathrm{p}=0.006)$, serum creatinine level $(\mathrm{p}=0.023)$, and TSP (hazard ratio: $0.159,95 \%$ confidence interval: $0.062-0.408, \mathrm{p}<0.001)$ predicted mortality independently.

Conclusion: TSP level predicted all-cause mortality independently in STEMI patients who underwent primary percutaneous coronary intervention during 8-year follow-up.

Keywords: ST-elevation myocardial infarction (STEMI), total serum protein (TSP), mortality, acute coronary syndrome, malnutrition

\section{Introduction}

ST-elevation myocardial infarction (STEMI) is still responsible for a large number of deaths globally, despite modern medical and interventional treatment options and early revascularization ${ }^{(1)}$. Therefore, determining the high-risk patient group is essential to provide earlier and intensive treatment modalities and for closer followup. Although many clinical factors that predict clinical outcomes in patients with STEMI have been identified, these scoring tools consisted of many clinical factors and were evaluated for less than 5 years. Besides, results were controversial ${ }^{(2)}$. Since clinical conditions such as myocardial fibrosis, ventricular remodeling, recurrent myocardial infarction (MI), ventricular dilatation, and arrhythmias, which are associated with mortality, occur in a long course, it can be asserted that longer follow-up could give more accurate results for mortality.

Serum albumin represents approximately half of the TSP and is the primary determinant of metabolic homeostasis. Albumin regulates microvascular permeability and plasma oncotic pressure, and has an antioxidant function. Hypoalbuminemia is a robust prognostic marker in patients with coronary artery disease $(\mathrm{CAD})^{(3,4)}$. In addition, an elevated level of globulin, referred to as gamma gap (difference between TSP and albumin), was associated with a worse outcome. Gamma gap-related mortality was demonstrated to be caused by inflammation. However, it has been evaluated with a scarce number of studies ${ }^{(5)}$.
On the other hand, total serum protein (TSP) is a composite indicator of immunity, nutrition, and metabolic balance by including albumin and globulin. Impaired nutritional status and immunity were shown to contribute to the prognosis of patients with $\mathrm{CAD}^{(6)}$. Therefore, TSP evaluation could be more beneficial to predict outcomes than albumin and globulin alone. Nevertheless, the prognostic value of TSP predicting mortality in STEMI has not been studied yet.

By considering the pivotal roles of TSP in cardiovascular diseases (CVDs), we postulated that a total protein could provide better mortality risk prediction above and beyond the known clinical risk factors, single protein, and established biomarkers. It was aimed to examine the role of TSP in predicting mortality in STEMI patients who underwent p-PCI during 8-year follow-up.

\section{Materials and Methods}

\section{Study Population}

This retrospective and observational study was carried with STEMI patients admitted to our clinic between March 2007 and May 2009. The sociodemographic data, past medical histories and detailed physical examinations of each patient were acquired at admission by experienced cardiologists and recorded in the database system of the hospital. Patients with $\geq 1 \mathrm{~mm}$ ST-elevation in consecutive leads related to one of the major coronary arteries' tertiaries on electrocardiography were accepted STEMI and delivered to angiography laboratory, immediately ${ }^{(1)}$. 
The STEMI diagnosis was confirmed by coronary angiogram. Institutional review board approved the study with 40465587-050.01.04-75 number on 09.04.2021 and the informed consent was obtained from patients or parents.

\section{Cardiovascular Risk Factors}

The diagnoses of hypertension and diabetes mellitus (DM) were established according to the current guidelines $^{(7)}$. The presence of familial history of CAD was defined as the development of atherosclerotic CVD or death from CVD in a first-degree relative (ie, parent or sibling) prior to age of 55 years for males or 65 years for females. Those who smoke at least 1 cigarette a day were defined as a regular smoker. Body mass index (BMI) was obtained by the weight/[height $\left.(\mathrm{cm})^{2}\right]$ formula.

\section{Laboratory Analysis}

Suitable samples for routine biochemistry, hemogram, creatinine kinase-MB (CK-MB), troponin, and inflammation markers were received from all patients at admission. Glucose and lipid parameters were evaluated after at least 8 hours of fasting and were determined using standard methods. CK-MB and troponin-I (Tn-I) measurements were repeated at 4-hour intervals. As the laboratory indicates Tn-I above $50 \mathrm{ng} / \mathrm{mL}$ as $>50 \mathrm{ng} / \mathrm{mL}$, it was included in the analysis as $50 \mathrm{ng} / \mathrm{mL}$. Blood samples obtained at the admission were immediately centrifuged, and the obtained serum specimen was awaited in a freezer at $-20{ }^{\circ} \mathrm{C}$. The amount of TSP was measured using colorimetric assay with the Beckman Synchron LX20.

\section{Coronary Angiography and Percutaneous Coronary Intervention}

Coronary angiography was conducted urgently using the Judkins technique in all patients. Left anterior descending and circumflex coronary arteries were viewed from at least four different angles and the right coronary artery from at least two different angles. The coronary artery with total occlusion was revascularized with the coronary balloon and/or stent immediately after imaging.
Thrombolysis in Myocardial Infarction (TIMI) flow was evaluated. In epicardial arteries with a diameter of $\geq 1.5$ $\mathrm{mm}$, any lesion that caused at least 50\% lumen narrowing compared to the closest segment was considered as significant stenosis. Intervention to the total occluded coronary artery at first angiogram was determined as the revascularization strategy. In case of a severe lesion other than the culprit lesion, elective PCI was planned. Patients were treated following the current guidelines and awaited in the coronary intensive care unit until stabilization was achieved $^{(8)}$.

\section{Transthoracic Echocardiography}

Detailed two-dimensional echocardiography was carried out in all patients before discharge and during routine follow-up by using Philips Epiq 7 systems (Andover, MA) and a 2.5-3.5-MHz transducer. Left atrial and ventricular dimensions and septal and posterior wall thickness were gained by M-mode echocardiography. In addition, conventional Doppler and Tissue Doppler parameters were obtained. Left ventricular ejection fraction (LVEF) was measured from left ventricular dimensions.

\section{Exclusion Criteria}

Patients complicated with PCI-related coronary dissection, coronary rupture, acute or hyperacute stent thrombosis, previous cardiovascular surgery with any indication, end-stage liver or kidney disease, malignancy, history of radiotherapy or chemotherapy, collagen vascular disease, history of cerebrovascular event, endocrine disorders, active or chronic inflammatory disease, moderate to severe valvular heart disease, pulmonary embolism, myocarditis, cardiogenic shock, previous cardiomyopathy and those with a BMI of 18.5 and/or below were excluded from the study.

\section{Clinical Follow-up and Primary Endpoint}

The primary endpoint of the study was all-cause mortality. The patients were followed for approximately 8 years. Mortality data were obtained by assessing the 
hospital medical records and national death database. In case mortality data could not be reached, direct phone calls to patients or their relatives were performed.

\section{Statistical Analysis}

SPSS 23.0 version software package (Chicago, IL) was employed to analyze the obtained data. A two-tailed p-value of $\leq 0.05$ was accepted as statistical significance. The Kolmogrov-Smirnov test and visual histograms were conducted to assess the normality assumption of variables. The homogeneity of variances was tested with the Levenes's test. While continuous variables were stated as mean \pm standard deviation, categorical variables were presented with percentages. The chi-square test was employed to compare the categorical groups. While the two-tailed Student's t-test was conducted for parameters that were normally distributed, continuous parameters that were non-normally distributed were analyzed with the Mann-Whitney $U$ test. The univariate regression analysis was carried out to assess the effects of the various variables on mortality. A p-value (2-tailed) of less than 0.05 was identified as statistical significance. The variables with unadjusted $\mathrm{p}<0.1$ were accepted to be confounding factors and included in the multivariable Cox logistic regression analysis. The predictive value of TSP for mortality was estimated by areas under the receiver operating characteristic (ROC) curve. Cumulative surveys of above and below the determined TSP value were compared by the Kaplan-Meier analysis.

\section{Results}

The mean age of a total 99 patients was $61 \pm 12.4$ years, and $82(82.8 \%)$ of them were male. Patients were separated into two groups according to the presence of mortality. The mortality group was older $(\mathrm{p}=0.05)$ and had a higher rate of $\mathrm{DM}(\mathrm{p}=0.02)$. Both groups exhibited similar characteristics in terms of gender $(\mathrm{p}=0.246)$, hypertension $(\mathrm{p}=0.456)$ and other demographic features (Table 1).

The time from the onset of the STEMI-related complaints to the percutaneous intervention was determined as the pain-to-balloon time, and although it did not reach statistical significance, it was found to be longer in the mortality group [270 (150-450) vs 330 (220-480), $\mathrm{p}=0.259]$. On the other hand, TIMI flow after revascularization $(\mathrm{p}=0.001)$ and LVEF $(45.4 \pm 10.5$ vs $38.6 \pm 6.9, \mathrm{p}=0.001$ ) were lower in the mortality group. Furthermore, serum creatinine $(0.93 \pm 0.22$ vs $1.1 \pm 0.46$ $\mathrm{mg} / \mathrm{dL}, \mathrm{p}=0.003)$ and fasting blood glucose [105 (92-122) vs 119 (97-176), $\mathrm{p}=0.008$ ] were higher in the mortality group. Although they were within the normal range in both groups, hemoglobin ( $14.2 \pm 1.5$ vs $13.2 \pm 1.3 \mathrm{~g} / \mathrm{dL}, \mathrm{p}=0.002)$, albumin ( $4.2 \pm 0.32$ vs $4.0 \pm 0.33 \mathrm{mg} / \mathrm{dL}, \mathrm{p}=0.014)$, and TSP (7.2 \pm 0.51 vs $6.8 \pm 0.45 \mathrm{mg} / \mathrm{dL}, \mathrm{p}<0.001)$ were lower in the mortality group (Table 1 ).

Backward multivariable Cox regression analysis demonstrated that DM [hazard ratio $(\mathrm{HR})=3.073,95 \%$ confidence interval $(\mathrm{CI})=1.361-6.931, \mathrm{p}=0.007]$, age $(\mathrm{HR}=1.045,95 \% \quad \mathrm{CI}=1.005-1.086, \quad \mathrm{p}=0.027), \quad \mathrm{LVEF}$ $(\mathrm{HR}=0.934, \quad 95 \% \quad \mathrm{CI}=0.890-0.981, \quad \mathrm{p}=0.006), \quad$ serum creatinine level $(\mathrm{HR}=3.025,95 \% \quad \mathrm{CI}:=1.162-7.877$, $\mathrm{p}=0.023)$, and $\mathrm{TSP}(\mathrm{HR}=0.159,95 \% \mathrm{CI}=0.062-0.408$, $\mathrm{p}<0.001$ ) were determined as independent predictors of mortality (Table 2).

ROC analysis showed that TSP value below 6.95 $\mathrm{mg} / \mathrm{dL}$ predicted mortality with $73.8 \%$ sensitivity and $69 \%$ specificity (AUC $=0.211, \mathrm{p}<0.001)$ (Figure 1). Additionally, the Kaplan-Meier curve showed that TSP $<6.95 \mathrm{mg} / \mathrm{dL}$ increased the risk of mortality starting from the early phase up to 8 years of follow-up (Figure 2).

\section{Discussion}

It was found that TSP, age, presence of DM, serum creatinine level, and LVEF were independent predictors of mortality after 8-year follow-up in STEMI patients who underwent p-PCI. The role of TSP in predicting mortality after long-term follow-up has not been studied previously.

TSP consists of albumin and globulin fraction, including enzymes, complements, and carrier proteins. These proteins are mainly responsible for inflammation, immunity, and nutritional balance ${ }^{(6,8)}$. C-reactive protein, pro-brain natriuretic peptide (pro-BNP), fibrinogen, and 
Table 1. Baseline characteristics of the study population.

\begin{tabular}{|c|c|c|c|c|}
\hline \multirow{2}{*}{ Variable } & $\begin{array}{l}\text { Mortality (-) } \\
(n=68)\end{array}$ & $\begin{array}{l}\text { Mortality (+) } \\
(n=31)\end{array}$ & $\begin{array}{l}\text { All patients } \\
(n=99)\end{array}$ & p-value \\
\hline & \multicolumn{4}{|c|}{ Demographic data } \\
\hline Age (years) & $58.4 \pm 11.5$ & $66.9 \pm 12.8$ & $61 \pm 12.4$ & 0.005 \\
\hline Gander (male), n (\%) & $58(85.3)$ & $24(77.4)$ & $82(82.8)$ & 0.246 \\
\hline Diabetes mellitus, n (\%) & $11(16.2)$ & $14(45.2)$ & $25(25.3)$ & 0.002 \\
\hline Hypertension, n (\%) & $24(35.3)$ & $12(38.7)$ & $36(36.4)$ & 0.456 \\
\hline Hyperlipidemia, n (\%) & $41(60.3)$ & $15(48.4)$ & $56(56.6)$ & 0.187 \\
\hline Current smoking, n (\%) & $30(44.1)$ & $8(25.8)$ & $38(38.4)$ & 0.183 \\
\hline BMI $\left(\mathrm{kg} / \mathrm{m}^{2}\right)$ & $27.5 \pm 3.9$ & $27.3 \pm 4.8$ & $27.5 \pm 4.1$ & 0.849 \\
\hline PBT (sec) & $270(150-450)$ & $330(220-480)$ & $270(172-450)$ & 0.259 \\
\hline Heart rate (beat/min) & $81(62-94)$ & $84(70-91)$ & $83(69-92)$ & 0.854 \\
\hline LV EF (\%) & $45.4 \pm 10.5$ & $38.6 \pm 6.9$ & $43.6 \pm 10.1$ & 0.001 \\
\hline \multicolumn{5}{|l|}{ Killip } \\
\hline $1, \mathrm{n}(\%)$ & $57(83.8)$ & $23(74.2)$ & $80(80.8)$ & \multirow{2}{*}{0.264} \\
\hline$\geq 2, \mathrm{n}(\%)$ & $11(16.2)$ & $8(25.8)$ & $19(19.2)$ & \\
\hline \multicolumn{5}{|l|}{ Laboratory data } \\
\hline Peak CK-MB (ng/uL) & $235(128-300)$ & $300(175-300)$ & 108 (94-139) & 0.253 \\
\hline Peak Troponin (ng/uL) & $45.2 \pm 10.8$ & $46.9 \pm 8.9$ & $45.7 \pm 10$ & 0.471 \\
\hline WBC $\left(10^{3} / \mu \mathrm{L}\right)$ & $11.06 \pm 3.9$ & $12.8 \pm 5.1$ & $11.5 \pm 4.3$ & 0.082 \\
\hline Hemoglobin (g/dL) & $14.2 \pm 1.5$ & $13.2 \pm 1.3$ & $13.9 \pm 1.5$ & 0.002 \\
\hline Creatinine (mg/dL) & $0.93 \pm 0.22$ & $1.1 \pm 0.46$ & $0.97 \pm 0.30$ & 0.003 \\
\hline Fasting glucose (mg/dL) & $105(92-122)$ & $119(97-176)$ & 108 (94-139) & 0.008 \\
\hline $\mathrm{HsCrp}(\mathrm{mg} / \mathrm{dL})$ & $0.39(0.29-1.11)$ & $0.76(0.210-1.48)$ & $0.43(0.25-1.28)$ & 0.093 \\
\hline Total Protein (mg/dL) & $7.2 \pm 0.51$ & $6.8 \pm 0.45$ & $7.1 \pm 0.5$ & $<0.001$ \\
\hline Albumin (mg/dL) & $4.2 \pm 0.32$ & $4.0 \pm 0.33$ & $4.1 \pm 0.33$ & 0.014 \\
\hline Type of MI (anterior), n (\%) & $30(44.1)$ & $18(58.1)$ & $48(48.5)$ & 0.202 \\
\hline \multicolumn{5}{|l|}{ Angiographic data } \\
\hline \multicolumn{5}{|l|}{ IRA count } \\
\hline 1 & $23(33.8)$ & $4(12.9)$ & $27(27.3)$ & \multirow{3}{*}{0.020} \\
\hline 2 & $25(36.8)$ & $12(38.7)$ & $37(37.4)$ & \\
\hline 3 & $20(29.4)$ & $15(48.7)$ & $35(35.4)$ & \\
\hline \multicolumn{5}{|l|}{ IRA } \\
\hline LAD & $31(45.6)$ & $18(58.1)$ & $49(49.5)$ & \multirow{3}{*}{0.389} \\
\hline RCA & $25(36.8)$ & $8(25.8)$ & $33(33.3)$ & \\
\hline$C X$ & $12(17.6)$ & $5(16.1)$ & $17(17.2)$ & \\
\hline \multicolumn{5}{|l|}{ Final TIMI flow, n (\%) } \\
\hline $0-1$ & $5(7.4)$ & $3(9.7)$ & $8(8.1)$ & \multirow{3}{*}{0.001} \\
\hline 2 & $8(11.8)$ & $14(45.2)$ & $22(22.2)$ & \\
\hline 3 & $55(80.9)$ & $14(45.2)$ & $69(69.7)$ & \\
\hline \multicolumn{5}{|l|}{ Medication at discharge } \\
\hline ASA, $n(\%)$ & $68(100)$ & $27(87.1)$ & $95(96)$ & 0.734 \\
\hline Clopidogrel, n (\%) & $68(100)$ & $29(98.3)$ & $97(98.9)$ & 0.987 \\
\hline CСB, n (\%) & $3(4.4)$ & $0(0)$ & $3(3)$ & 0.405 \\
\hline BB, n (\%) & $65(95.6)$ & $24(77.4)$ & $89(89.9)$ & 0.121 \\
\hline Statin, n (\%) & $66(97.1)$ & $27(87.1)$ & 93 (93.9) & 0.747 \\
\hline ACEI/ARB n (\%) & $65(95.5)$ & $29(93.5)$ & $94(94.9)$ & 0.153 \\
\hline
\end{tabular}

BMI: Body mass index, PBT: Paint o balloon time, LVEF: Left ventricle ejection fraction, CK-MB: Creatine kinase, WBC: White blood count, HsCrp: High sensitive C-reactive protein, MI: Myocardial Infarction, IRA: Infarct related artery, LAD: Left anterior descending artery, RCA: Right coronary artery, CX: Circumflex artery, ASA: Acetyl salicylic acid, CCB: Calcium channel blocker, BB: Beta blocker, ACEl: Angiotensin-converting enzyme inhibitor, ARB: Angiotensin receptor blockers, $n$ : Number 


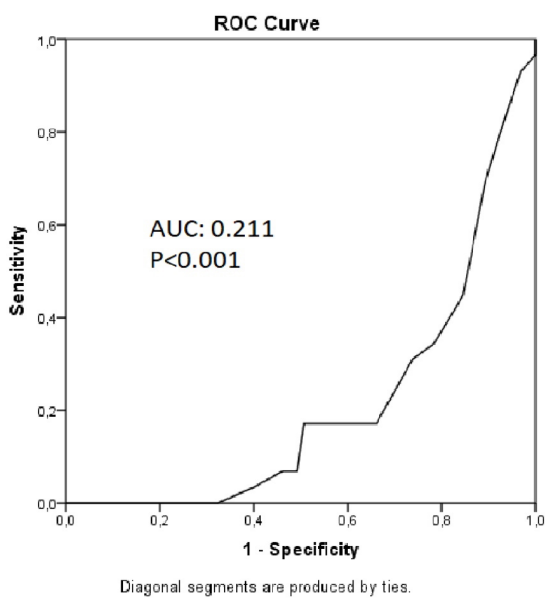

Figure 1. TSP $<6.95 \mathrm{mg} / \mathrm{dL}$ predicted mortality with $73.8 \%$ sensitivity and $69 \%$ specificity

TSP: Total serum protein, ROC: Receiver operating characteristic, AUC: Area under the curve

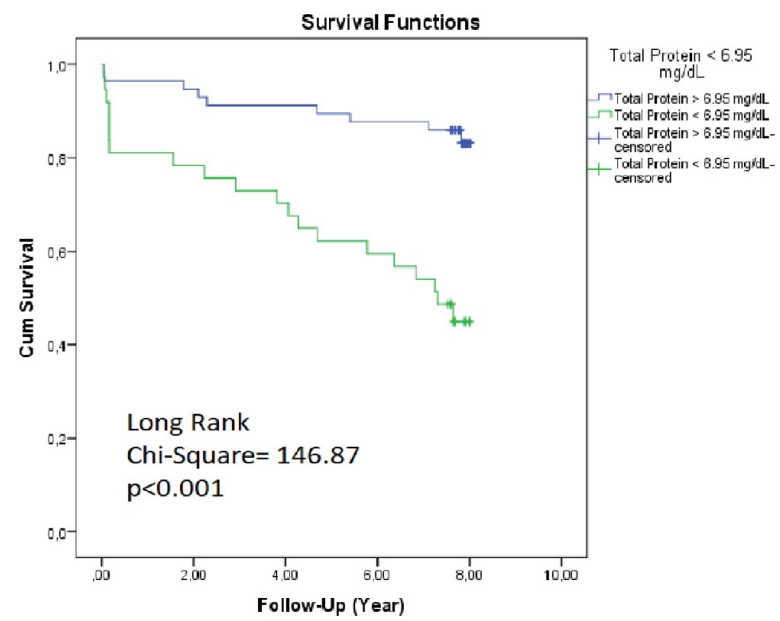

Figure 2. Kaplan-Meier curve showing TSP $<6.95 \mathrm{mg} / \mathrm{dL}$ increases the risk of mortality for 8 years of follow-up TSP: Total serum protein, Cum: Cumulative

Table 2. Regression analysis for mortality predictors

\begin{tabular}{|c|c|c|c|c|c|c|}
\hline \multirow[b]{2}{*}{ Variable } & \multicolumn{3}{|c|}{ Univariable } & \multicolumn{3}{|c|}{ Multivariable } \\
\hline & OR & $95 \% \mathrm{Cl}$ & $p$-value & OR & $95 \% \mathrm{Cl}$ & $p$-value \\
\hline Diabetes mellitus & 3.240 & $1.593-6.591$ & 0.001 & 3.073 & $1.361-6.931$ & 0.007 \\
\hline Fasting glucose & 1.008 & $1.004-1.013$ & $<0.001$ & & & \\
\hline Age & 1.055 & $1.021-1.090$ & 0.001 & 1.045 & $1.005-1.086$ & 0.027 \\
\hline LVEF & 0.933 & $0.895-0.972$ & 0.001 & 0.934 & $0.890-0.981$ & 0.006 \\
\hline Hemoglobin & 1.103 & $1.082-1.124$ & 0.018 & & & \\
\hline Serum creatinine & 4.716 & $2.097-10.604$ & $<0.001$ & 3.025 & $1.162-7.877$ & 0.023 \\
\hline Total Protein & 0.246 & $0.113-0.535$ & $<0.001$ & 0.159 & $0.062-0.408$ & $<0.001$ \\
\hline Albumin & 0.275 & $0.107-0.702$ & 0.007 & & & \\
\hline IRA count & 1.818 & $1.120-2.951$ & 0.016 & & & \\
\hline Final TIMI flow & 0.515 & $0.330-0.802$ & 0.003 & & & \\
\hline
\end{tabular}

albumin are also established proteins among TSP, which was shown to play an active role in CVDs. These proteins have also been shown to be associated with mortality in MI patients ${ }^{(9)}$. Albumin constitutes the major component, and its effects on the cardiovascular system are responsible for most of the functions of $\mathrm{TSP}^{(4)}$. On the other hand, few studies were conducted with globulin or gamma gap evaluating their effects on prognosis. Low globulin is also shown to be associated with the atherosclerosis development starting from the fatty streaking phase, and gamma globulin therapy prevents the progression of atherosclerosis ${ }^{(5)}$. On the contrary, an elevated amount of globulin and its equivalent, albumin to globulin ratio, is related to higher mortality rate. We found that albumin 
could not predict mortality independently. Our results have supported the fact that a decrease in globulin level increases mortality rate. Thus, it can be postulated that the globulin has more effects than known and plays an active role in the post-MI course. However, the predictive role of the alteration in the albumin level seems superior to that of the other proteins, yet further prospective studies are needed.

Albumin affects the cardiovascular system by regulating plasma oncotic pressure, microvascular permeability, maintenance of acid-base balance, and the transport of many endogenous and exogenous molecules ${ }^{(3,9)}$. Hypoalbuminemia was demonstrated to be associated with elevated adverse outcomes in various clinical settings such as heart failure, stroke, peripheral artery disease, renal failure, and $\mathrm{CAD}^{(10,11)}$. Besides, albumin has a substantial role in the atherosclerotic process in line with following mechanisms: (I) Heparin-like platelet inhibition function, (II) Prostacyclin (PGI2), which is a natural vasodilator and platelet aggregation inhibitor and functions upon albumin concentration, and (III) Homocysteine, which is known to induce atherosclerosis and acts by binding to albumin. Therefore, a reduction in the albumin level diminishes the anti-atherosclerotic effects of PGI2 and homocysteine. Furthermore, hypoalbuminemia is shown to induce the new $\mathrm{MI}$ in patients with $\mathrm{CAD}^{(12)}$.

Albumin is a highly antioxidant component of TSP. STEMI causes cellular dysfunction by creating reactive oxygen species (ROS) followed by oxidative stress, and albumin absorbs these ROS radicals ${ }^{(13)}$. In addition, bilirubin and nitric oxide, the antioxidant molecules, function depending on albumin. The decreased albumin is associated with elevated oxidative stress and consequent myocardial damage ${ }^{(14)}$. Thus, a higher mortality rate, when the TSP level is lower, can be explained by decreased antioxidant activity. Meanwhile, albumin is also a negative acute-phase reactant inversely correlated with inflammation, and it is a marker of a potency of inflammatory response secondary to STEMI ${ }^{(15)}$.

It is important to state that albumin has a substantial role in the pharmacokinetics of cardiovascular drugs, especially clopidogrel and aspirin. Previous experimental studies found that low albumin level was associated with aspirin resistance in CAD patients ${ }^{(16,17)}$. Correspondingly, it can be asserted that lower TSP level is associated with decreased cardiovascular drug efficacy. Further studies regarding the relationship of TSP with cardiovascular pharmacology are needed.

Immune response initiates to repair myocardial damage just after MI. Immunoglobulins, cytokines, and complements have a crucial role in this immune response. It is established that patients with suppressed immunity are more vulnerable to future adverse clinical events after $\mathrm{MI}^{(5,18)}$. Hence, it can be asserted that lower TSP is related to an inadequate immune response, which is associated with increased mortality. On the other hand, malnutrition was shown to be associated with poor outcomes in patients with malignancy, undergoing surgery, and with CAD in several studies $^{(19,20)}$. For that purpose, albumin and albumin-based indices such as prognostic nutritional index, nutritional risk index, and mini nutritional assessment were used to predict outcomes in various clinical settings ${ }^{(21)}$. With this respect, it could be argued that TSP is a better malnutrition marker than albumin or globulin alone.

\section{Study Limitations}

This is a single-center study with a limited number of patients. The effect of albumin level on mortality through possible reduction of drug efficacy was ignored. Secondly, the changes in patient-related cardiovascular risk factors, medications and device therapies, follow-up centers, and new interventions may affect adverse event rates over decades. Non-cardiovascular mortality may be higher than expected in 8 years.

\section{Conclusion}

TSP is an independent predictor of all-cause mortality in patients with STEMI, who underwent p-PCI, during 8 -year follow-up. It could be possible to improve the mortality rate by regulating the total protein level in these patients. 


\section{Ethics}

Ethics Committee Approval: Recep Tayyip Erdoğan University Ethics Committee has approved the study with 40465587-050.01.04-75 number on 09.04.2021 and the informed consent were obtained from patient or parents.

Informed Consent: Informed consent were obtained from patient or parents.

Peer-review: Externally peer-reviewed.

\section{Authorship Contributions}

Concept: A.S.Y., F.K., Design: A.S.Y., F.K., Data Collection or Processing: A.S.Y., M.Ç., Analysis or Interpretation: A.S.Y., A.G.Ö., M.Ç., Literature Search: A.S.Y., M.Ç., Statistical Analysis: M.Ç., F.K., Writing: A.S.Y., Critical review: A.G.Ö., F.K.

Conflict of Interest: The authors declare that they have no conflict of interest.

Financial Disclosure: This research received no grant from any funding agency in the public, commercial or notfor-profit sectors.

\section{References}

1. Ibanez B, James S, Agewall S, et al. 2017 ESC Guidelines for the management of acute myocardial infarction in patients presenting with STsegment elevation: The Task Force for the management of acute myocardial infarction in patients presenting with ST-segment elevation of the European Society of Cardiology (ESC). Eur Heart J 2018;39:119-77.

2. Lev EI, Kornowski R, Vaknin-Assa H, et al. Comparison of the predictive value of four different risk scores for outcomes of patients with STelevation acute myocardial infarction undergoing primary percutaneous coronary intervention. Am J Cardiol 2008;102:6-11.

3. Chien SC, Chen CY, Lin CF, Yeh HI. Critical appraisal of the role of serum albumin in cardiovascular disease. Biomark Res 2017;5:31.

4. Nelson JJ, Liao D, Sharrett AR, et al. Serum albumin level as a predictor of incident coronary heart disease: the Atherosclerosis Risk in Communities (ARIC) study. Am J Epidemiol 2000;151:468-77.

5. Loprinzi PD, Addoh O. The gamma gap and all-cause mortality risk: considerations of physical activity. Int J Clin Pract 2016;70:625-9.

6. Wang M, Liu J, Ma C, et al. Synergistic association of serum albumin and globulin with coronary heart disease. J Atheroscler Thromb 2012;19:61932 .
7. de Boer IH, Bangalore S, Benetos A, et al. Diabetes and Hypertension: A Position Statement by the American Diabetes Association. Diabetes Care 2017;40:1273-84.

8. Collet JP, Thiele H, Barbato E, et al. 2020 ESC Guidelines for the management of acute coronary syndromes in patients presenting without persistent ST-segment elevation. Eur Heart J 2021;42:1289-1367.

9. Quinlan GJ, Martin GS, Evans TW. Albumin: biochemical properties and therapeutic potential. Hepatology 2005;41:1211-9.

10. Djoussé L, Rothman KJ, Cupples LA, Levy D, Ellison RC. Serum albumin and risk of myocardial infarction and all-cause mortality in the Framingham Offspring Study. Circulation 2002;106:2919-24.

11. Oduncu V, Erkol A, Karabay CY, et al. The prognostic value of serum albumin levels on admission in patients with acute ST-segment elevation myocardial infarction undergoing a primary percutaneous coronary intervention. Coron Artery Dis 2013;24:88-94.

12. Akirov A, Masri-Iraqi H, Atamna A, Shimon I. Low Albumin Levels Are Associated with Mortality Risk in Hospitalized Patients. Am J Med 2017;130:1465.e11-1465.e19.

13. Berg K, Jynge P, Bjerve K, Skarra S, Basu S, Wiseth R. Oxidative stress and inflammatory response during and following coronary interventions for acute myocardial infarction. Free Radic Res 2005;39:629-36.

14. Taverna M, Marie AL, Mira JP, Guidet B. Specific antioxidant properties of human serum albumin. Ann Intensive Care 2013;3:4.

15. Cirakoglu OF, Aslan AO, Yilmaz AS, Şahin S, Akyüz AR. Association Between C-Reactive Protein to Albumin Ratio and Left Ventricular Thrombus Formation Following Acute Anterior Myocardial Infarction. Angiology 2020;71:804-11.

16. Niaei N, Hasanzadeh M, Shadjou N. Molecular interaction of some cardiovascular drugs with human serum albumin at physiological-like conditions: A new approach. J Mol Recognit 2018;31:e2715.

17. Ganesan S, Williams C, Maslen CL, Cherala G. Clopidogrel variability: role of plasma protein binding alterations. Br J Clin Pharmacol 2013;75:146877.

18. Swirski FK, Nahrendorf M. Cardioimmunology: the immune system in cardiac homeostasis and disease. Nat Rev Immunol 2018;18:733-44.

19. Bharadwaj S, Ginoya S, Tandon P, et al. Malnutrition: laboratory markers vs nutritional assessment. Gastroenterol Rep (Oxf) 2016;4:272-80.

20. Chan R, Leung J, Woo J. High Protein Intake Is Associated with Lower Risk of All-Cause Mortality in Community-Dwelling Chinese Older Men and Women. J Nutr Health Aging 2019;23:987-96.

21. Chen QJ, Qu HJ, Li DZ, et al. Prognostic nutritional index predicts clinical outcome in patients with acute ST-segment elevation myocardial infarction undergoing primary percutaneous coronary intervention. Sci Rep 2017;7:3285. 